\title{
Viscoelastic tuning of fibre Bragg gratings during regeneration
}

John Canning, Sharoui Gao, Kevin Cook, Arnaud Loubert, Matthieu Lancry

John Canning, Sharoui Gao, Kevin Cook, Arnaud Loubert, Matthieu Lancry, "Viscoelastic tuning of fibre Bragg gratings during regeneration," Proc. SPIE 9157, 23rd International Conference on Optical Fibre Sensors, 9157AJ (2 June 2014); doi: 10.1117/12.2058990

SPIE Event: OFS2014 23rd International Conference on Optical Fiber Sensors, 2014, Santander, Spain 


\title{
Viscoelastic tuning of fibre Bragg gratings during regeneration
}

\author{
John Canning* ${ }^{\mathrm{a}}$, Shaorui Gao ${ }^{\mathrm{a}, \mathrm{b}}$, Kevin Cook $^{\mathrm{a}}$, Arnaud Loubert ${ }^{\mathrm{a}, \mathrm{c}}$, Matthieu Lancry ${ }^{\mathrm{c}}$ \\ ${ }^{a}$ interdisciplinary Photonics Laboratories (iPL), School of Chemistry, The University of Sydney, NSW 2006, Australia \\ ${ }^{\mathrm{b}}$ Centre for Optical and Electromagnetic Research, State Key Laboratory of Modern Optical Instrumentation, Zhejiang \\ University, Hangzhou 310058, China \\ ${ }^{c}$ ICMMO, UMR CNRS-UPS 8182, Bâtiment 410, Université Paris Sud 11, 91405 Orsay, France
}

\begin{abstract}
Regenerating at constant temperature under load allows inelastic changes in glass that can be exploited to tune the Bragg wavelength of a filter to any arbitrary spectral position. We have reported $>20 \mathrm{~nm}$ of tuning of a $1 \mathrm{~cm}$ grating with no limit in sight. Further, regenerating under a temperature profile allows complex spectral shaping of the grating profile. As an example, we have reported broadband chirping by more than $\Delta \lambda>9 \mathrm{~nm}$ over $1 \mathrm{~cm}$ using this approach. High temperature viscoelastic tuning therefore offers a simple and powerful low cost way of tuning the properties of fibre Bragg gratings using a single phase mask.
\end{abstract}

Keywords: Regeneration, gratings, fibre sensors, spectral coding, tuning, chirped, broadband filters, FBG

\section{INTRODUCTION}

Regenerated fibre Bragg gratings [1] have excellent performance in terms of ultra-high temperature survival ability and represent the penultimate high temperature silica sensor [2] capable of long term performance if packaged appropriately [3]. They require little more than a phase mask and direct writing followed by high temperature annealing in the presence of a strain-inducing gas, commonly hydrogen. The strength is proportional to the seed grating strength - the stronger the seed, the stronger the subsequent local modulation. Thus photosensitive fibres produce strong seed gratings, discriminating more the periodic grating regions. A reasonably optimised process can achieve index modulation ratios exceeding $20 \%$, depending on the numerical aperture of the fibre.

In order to maintain the simplicity and relative low cost of the direct writing process through a single phase mask, simple tuning of the period is desirable. In general, all things being constant and with no chemical diffusion involved, regeneration preserves the phase information of the seed grating [4], suggesting total control is possible in a way that is distinct from past work requiring multiple phase masks, complex phase masks or complicated and less stable interferometric writing setups (though all these can be integrated with such a process).

Regeneration occurs in the viscoelastic softening regime of the silica cladding - this means we can in principle arbitrarily tune and shape the regeneration profile with high precision. Here we realize such potential.

\section{VISCOELASTIC TUNING}

After $1000{ }^{\circ} \mathrm{C}$, an optical fibre whilst maintaining its integrity becomes malleable under load [5,6]. This issue can be a practical applications problem if packaging is not done correctly such there is no permanent strain but otherwise it offers a novel tuning route for high temperature gratings. The elongation under load will be inelastic and is an intrinsic property dictated by the amorphous silica cladding. The wavelength shift $\Delta \lambda_{\mathrm{B}}$ during elongation, under constant temperature and load along the grating, can be described as:

$$
\frac{\Delta \lambda_{B}}{\lambda_{B}}=\left(1-p_{e}\right) \varepsilon=\left(1-p_{e}\right) \frac{\Delta \Lambda}{\Lambda}=\left(1-p_{e}\right) \frac{\Delta L}{L}
$$

where $p_{e}$ is the strain optic coefficient, $\varepsilon$ is the strain, $\Lambda$ is the pitch of the grating, $L$ is the length of the section of fibre that is elongated, $\Delta L$ is the variation of the length of this section under local heating. $\Delta \lambda_{\mathrm{B}}$ shifts linearly with time. The wavelength tuning rate, or stretching coefficient, $r=\mathrm{d} \Delta \lambda_{\mathrm{B}} / \mathrm{d} t$ is linear with load [2] but exponentially varying with temperature in the viscoelastic regime from 1000 to $1150^{\circ} \mathrm{C}[6]$ :

$$
r(T)=A \exp (B / T)
$$


where $A$ and $B$ are scaling factors, $T$ is in kelvin. Thus there are two parameters that can be tuned here: one is the temperature itself and the other is the temperature profile along a seed grating.

\section{WIDE SPECTRAL SPAN GRATING ARRAYS FROM ONE PHASE MASK}

To enable quasi-distributed fibre sensing for high-temperature, harsh environmental applications, regenerated gratings need to be multiplexed into a grating array. Here, we present a novel method for producing regenerated grating arrays based on high-temperature strain-tuning.

Three identical seed gratings $(L=1 \mathrm{~cm}, \Delta L=6 \mathrm{~cm})$ were inscribed into a single $\mathrm{H}_{2}$ loaded $(\sim 180 \mathrm{~atm})$, B - codoped germanosilicate fibre using direct writing with an $\mathrm{ArF}$ laser through a $10 \mathrm{~mm}$ phase mask. The central wavelength and reflectivity of these seed gratings are $\lambda_{\mathrm{B}}=1547.7 \mathrm{~nm}$ and $R_{s}=99.999 \%$ respectively.

A high-temperature oven was used for annealing and simultaneously regenerating the grating array $\left(T \sim 850{ }^{\circ} \mathrm{C}, t \sim 30\right.$ min). The gratings decayed below the noise floor before regenerating and saturating at a peak reflection $R_{\mathrm{r}} \sim 10 \%$. The wavelengths of all three regenerated gratings are $\lambda_{\mathrm{B}}=1546.2 \mathrm{~nm}$. The gratings were then individually tuned under load $(6 \mathrm{~g})$.

The thermal processing recipe (Figure 1 (a)) involves heating to $850{ }^{\circ} \mathrm{C}$ within $60 \mathrm{~min}$, kept constant $\sim 10$ min and then increased to $1100{ }^{\circ} \mathrm{C}$ within $30 \mathrm{~min}$. There is initially an elastic and linear increase in wavelength with temperature consistent with the temperature dependent refractive index of glass. After $1000{ }^{\circ} \mathrm{C}$, the fibre cladding enters the soft viscous regime - under load, the observed shift accelerates with temperature consistent with inelastic stretching of the fibre. For the first grating, heating was stopped when $\lambda_{\mathrm{B}}=1585.8 \mathrm{~nm}$. Upon cooling this dropped to $\lambda_{\mathrm{B}}=1569.4 \mathrm{~nm}$. The second regenerated grating was similarly tuned to $1557.7 \mathrm{~nm}$ and after cooling, the regenerated grating array had a wavelength spacing of $\Delta \lambda_{\mathrm{B}}=11.5 \mathrm{~nm}$ over a $23 \mathrm{~nm}$ span, shown in Figure 1(b).

(a)

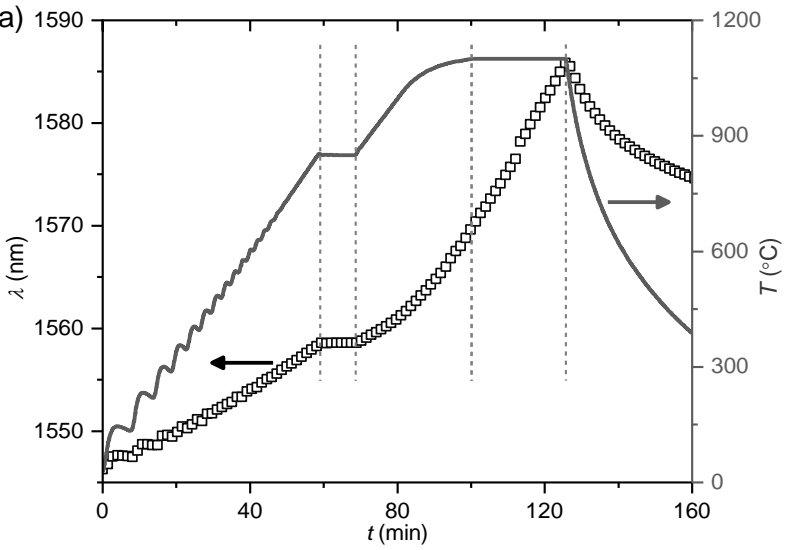

(b)

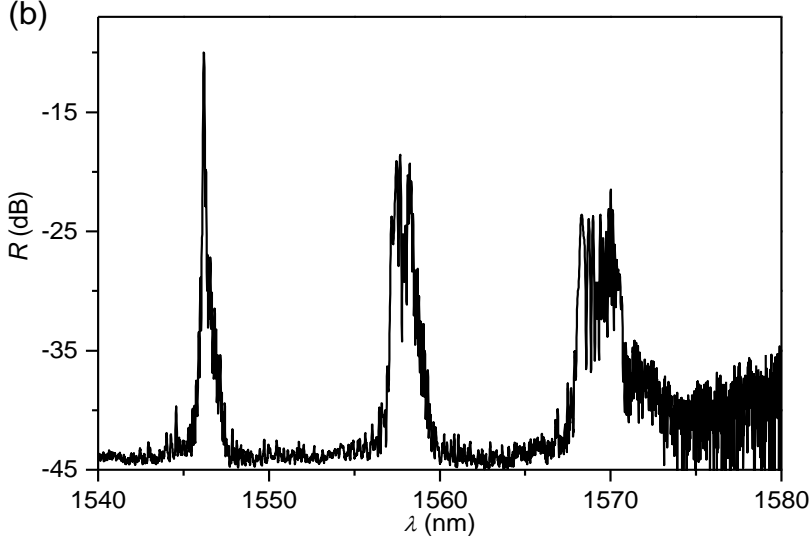

Figure 1. Regenerating arrays: (a) Evolution of the Bragg wavelength during tuning; (b) Final grating array spectrum at room temperature. The increased signal-to-noise at long wavelengths shows where the EDFA used to probe the spectrum drops off.

\section{ULTRABROADBAND FILTERS AND POTENTIAL DISPERSION COMPENSATORS FROM A UNIFORM MASK}

If the temperature profile along the regenerated grating is not constant any more but instead has a profile as a function of position along the grating, where $T=T(z)$, then:

$$
r(z)=r(T(\mathrm{z}), F)
$$

where $F$ is the force produced by the load, assuming a constant load. Therefore, Eq. (1) can be rewritten as:

$$
\frac{\Delta \lambda_{B}(r(z), t)}{\lambda_{B}}=\left(1-p_{e}\right) \frac{\Delta \Lambda(r(z), t)}{\Lambda}=\left(1-p_{e}\right) \frac{\Delta L(r(z), t)}{L}
$$

where $t$ is time lapse. Therefore, it is possible to employ a particular temperature profile $T(z)$ and wavelength tuning rate $r(z)$ to customize the profile of the grating period. 
For this work we review our recent report on chirped gratings [7]. Broadband components are increasingly of interest in many sensor applications, whether they be for interrogators, large spectral filters or so on. Chirped regenerated fibre gratings offer the possibility of future high-temperature sensor filters and even high temperature communication components such as dispersion compensators that can survive extreme conditions such as fires and other heat-escalating industrial and conflict environments. They can also be used in next generation of air and space craft and instrumentation.

A seed grating $(L=1 \mathrm{~cm})$, was inscribed in $\mathrm{H}_{2}$ loaded ( $\left.180 \mathrm{~atm}\right)$ B-codoped germanosilicate fibre using direct writing with an $\mathrm{ArF}$ laser through a $10 \mathrm{~mm}$ phase mask. The central wavelength and reflectivity of the seed grating is $\lambda_{\mathrm{B}}=1547.7 \mathrm{~nm}$ and $R_{s}=99.999 \%$ respectively.

The same high-temperature heater used for tuning the array was used $\left(T \sim 850{ }^{\circ} \mathrm{C}, t \sim 30 \mathrm{~min}\right)$. Over the heating period, the grating decayed completely before regenerating and saturating at a peak reflection $\operatorname{Rr} \sim 20 \%$. As shown above, under a longitudinal load, the fibre can be physically elongated depending on the heating temperature, load and duration. If the applied temperature is equal along the grating there is no change in the grating spatial profile and therefore no change in the spectrum other than a permanent shift to longer wavelengths in $\lambda_{B}$.

In this experiment, one side of the quadratic temperature distribution of the heater is exploited to generate a gradient viscoelasticity along the grating. When there is an applied load $(6 \mathrm{~g})$ the strain becomes similarly chirped which translates to a chirp (quadratic) in grating pitch. As shown in Figure 2, the regenerated grating was heated to $T=1000{ }^{\circ} \mathrm{C}$ within $t=79 \mathrm{~min}$. At this point, the regenerated grating is showing a slight chirping with the load. At $T=1120{ }^{\circ} \mathrm{C}$, the glass is softened further so chirping is visibly much more rapid. Strain tuning is stopped after $t=136$ min when the grating is cooled back down to room temperature. The grating bandwidth (measured at 10dB) has been broadened to $\Delta \lambda_{\mathrm{B}}$ $=9.8 \mathrm{~nm}$.
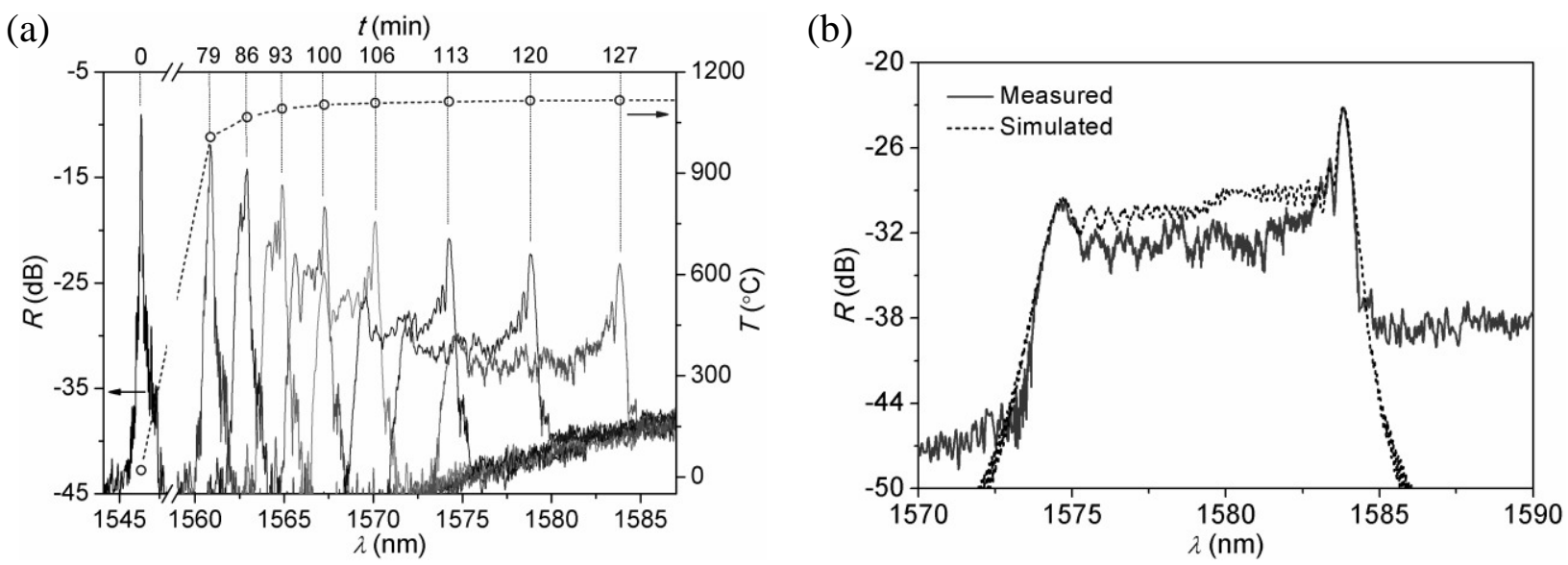

Figure 2. (a) Spectral evolution of the grating during the tuning process under load and temperature gradient (solid curves). Temperature recipe for tuning is also shown (circle dash); (b) Final spectrum at $t=127$ min and simulation based on temperature profile used.

\section{CONCLUSIONS}

We have demonstrated how a simple approach based on high temperature tuning in the viscoelastic regime of silica enables the tuning and fabrication of more complex arrays and filters from the same, simple uniform phase mask. The Bragg wavelength can potentially be tuned arbitrarily across all wavelengths and its profile tailored using an appropriate thermal gradient under applied load. These components retain the benefits of regeneration enabling the potential of complex components for very high temperature sensing and communications within extreme or stressed environments, including off-world.

\section{REFERENCES}

[1] Bandyopadhyay, S., Canning, J., Stevenson, M. and Cook, K., "Ultra-high temperature regenerated gratings in boron codoped germanosilicate optical fibre using 193nm”, Opt. Lett., 33(16), 1917-1919, (2008). 
[2] Canning, J., Stevenson, M., Bandyopadhyay, S. and Cook, K., "Extreme silica optical fibre gratings", Sensors 8 , 6448 (2008).

[3] Laffont, G., Cotillard, R. and Ferdinand, P., "9000 hours-long high temperature annealing of regenerated fiber Bragg gratings", in Fifth European Workshop on Optical Fibre Sensors, (International Society for Optics and Photonics, SPIE 2013), 87941X.

[4] Canning, J., Bandyopadhyay, S., Stevenson, M., Biswas, P., Fenton, J. and Aslund, M., "Regenerated gratings", J. Euro. Opt. Soc., Rapid Publications, 4, 09052 (2009).

[5] Wang,T., Shao, L.-Y., Canning, J. and Cook, K., "Regeneration of fiber Bragg gratings under strain," App. Opt. 52, 2080-2085 (2013).

[6] Shao, L. Y., Canning, J., Wang, T., Cook, K. and Tam, H. Y., "Viscosity of silica optical fibres characterized using regenerated gratings," Acta Materialia 61, 6071-6081 (2013).

[7] Gao, S., Canning, J. and Cook, K., "Ultra-high temperature chirped fiber Bragg gratings produced by gradient stretching of viscoelastic silica," Opt. Lett. 38 (24), 5397-5400 (2013). 